\title{
Analysis of the numerical results of the melting paraffin corks, ensuring the safe operation of the pipeline using a mobile source of electromagnetic radiation
}

\author{
Vera Titarenko, ${ }^{1, *}$ \\ ${ }^{1}$ Moscow Aviation Institute (National Research University), Moscow, Russian Federation
}

\begin{abstract}
For the safe operation of the pipeline, ensuring its smooth operation was conducted analysis of the results of numerical simulation of melting of paraffin jams with using a mobile source of electromagnetic radiation. Carried out varying different parameters of the radiation source, the peculiarities of its movement, the nature of the change of geometry of the boundary of melting of paraffin plugs under different external conditions
\end{abstract}

Reliable operation of pipelines for the transport of oil and gas condensate products in conditions favorable for the formation of crystalline hydrate and asphalt paraffin plugs requires the development of new methods for destroying such jams, as well as creating ways to prevent their formation. To eliminate paraffin plugs in real oil pipelines, it was proposed [1] to use a mobile source of electromagnetic radiation, called the "electromagnetic mole", in analogy with the "mechanical mole" used in the oil and gas industry for mechanical cleaning of pipelines from paraffin or gas hydrate plugs. The RF power of the electromagnetic wave is absorbed on the inner surface of the pipeline. Treatment of production wells is carried out while moving the source of electromagnetic radiation in the pipe in the longitudinal direction. This source produces an impact on the transported products, hindering the adhesion of the resultant particles of wax, they cannot Deposit on pipe walls and must be carried away by the fluid flow. Using the source of electromagnetic radiation, the turnaround time required cleaning piping (oil) wire increases due to the decrease of crystallization of paraffin's.

The speed of movement of the source was determined by the speed of movement of the boundary between liquid and solid phases in the melting process of asphalt-paraffin deposits under the influence of high-frequency electromagnetic radiation. Paraffin corks in the pipeline will be considered as a cylindrical metallic waveguide completely filled with dielectric. The electromagnetic radiation source located in the plane $\mathrm{z}=0$ at $\mathrm{t}=0$. The damping of the electromagnetic wave is caused by volume losses in the dielectric corks and surface losses in the metal walls of a cylindrical waveguide due to the imperfection of the conductivity of its surface The process of heating and melting asphalt-paraffin deposits under the influence of high-frequency electromagnetic radiation was studied on the basis of heat conduction equations with a given external heat source, which moves in the pipe in the longitudinal direction so that the minimum longitudinal distance from it to the surface phase transition was permanent. Considered axisymmetric problem, equation of heat conduction is written in a General form without explicit allocation of the phase [2, 3]:

$$
\rho c_{T} \frac{\partial T}{\partial t}=\frac{1}{r} \frac{\partial}{\partial r}\left(\lambda r \frac{\partial T}{\partial r}\right)+\frac{\partial}{\partial z}\left(\lambda \frac{\partial T}{\partial z}\right)+Q(r, z, t)
$$

where $\rho$ - density of high-paraffin oil, $c_{T}$ - its specific heat $\lambda$ - thermal conductivity; $Q(r, z, t)$ - the power density of volumetric heat generation, like the thermal conductivity, was considered temperature-independent, and the heat capacity at the phase transition temperature of $T_{S}$, has $\delta$ - shaped feature: $c_{T}=c_{0}+L \delta\left(T-T_{S}\right)$, where $L$ is the latent heat of the phase transition, $\delta(T-$ $\left.T_{S}\right)$ - Delta function of Dirac.

The power density of volumetric heat release takes into account the displacement of the source of electromagnetic radiation according to the law $z=z_{0}(t)$.

$$
\begin{gathered}
Q=Q_{0} \Theta\left(z-z_{0}(t)\right) \exp \left(-\alpha\left(z-z_{0}(t)\right)\right) \\
\Theta\left(z-z_{0}\right)= \begin{cases}1, & z \geq z_{0} \\
0, & z<z_{0}\end{cases}
\end{gathered}
$$

$\alpha$ - power attenuation factor of high frequency electromagnetic wave

The heat equation is complemented by boundary conditions.

1) At the end of the corks at $z=0$, given in the form of Newtonian convective heat transfer:

$$
\left.\lambda \frac{\partial T}{\partial z}\right|_{z=0}=\varkappa_{1}\left[T(r, 0, t)-T_{0}\right]
$$

where $T_{0}-$ is the ambient temperature and initial

\footnotetext{
Corresponding author: vera xmel@mail.ru
} 
temperature of the paraffin corks, $x_{1}$ - is the heat transfer coefficient.

2) At the remote end of the corks $z=H$ there is no heat transfer:

$$
\left.\lambda \frac{\partial T}{\partial z}\right|_{z=H}=0
$$

3) On the lateral surface of the cylinder $r=R$ the boundary condition is also written as a convective heat transfer law, with a different heat transfer coefficient taking into account the heat release due to the absorption of an electromagnetic wave on the surface of the metal pipeline:

$$
\left.\lambda \frac{\partial T}{\partial r}\right|_{r=R}=x\left[T(R, z, t)-T_{0}\right]-q(z, t)
$$

where $x=N u \cdot \lambda / R$ - the coefficient of heat exchange with the external environment, $N u$ is the Nussle number.

For accuracy control used the law of conservation of energy, which immediately follows from the heat conduction equations with boundary conditions:

$$
\begin{gathered}
\lambda \rho \frac{\partial}{\partial t} \int d V \int_{T_{0}}^{T} c\left(T^{\prime}\right) d T^{\prime}=P\left[1-\exp \left(-\alpha\left(H-z_{0}(t)\right)\right)\right]- \\
-2 \pi \varkappa_{1} \int_{0}^{R} d r r\left[T(r, z=0)-T_{0}\right]-2 \pi \varkappa R \int_{0}^{H} d z\left[T(r=R, z)-T_{0}\right]
\end{gathered}
$$

The law of displacement $\mathrm{z} 0(\mathrm{t})$ of a moving source of electromagnetic radiation, necessary for closing the system of equations determining the process of liquidation of a paraffin plug, was defined as the law of motion of the interface between the liquid and solid phases by the same differential equation of heat conductivity.

Numerical simulation of melting process of paraffin plugs by a moving source of electromagnetic radiation by a wave of the TEM0n type is carried out, which studies various solutions of the heat equation with a movable partition boundary phases by the pass-through account method, using a scheme of alternating directions The calculation was carried out in the operating system MS Windows $\mathrm{C}++$, using libraries: Qt, QCustomPlot, Eigen, Boost. Calculated temperature field displayed in the right part of the window. Because the problem is cylindrically symmetric, it displays only half of the pipeline, and the axis of symmetry is on the left. The position of the radiation source at the process of calculation is dynamically updated and the melting boundary of the paraffin corks is dynamically drawn.

The calculations were limited to consideration of the $E_{01}$ wave. The cut-off frequency of $E_{01}$ waves for the considered cylindrical waveguide is equal to $f_{0} \sim 0.97$.

$10^{9} \mathrm{~Hz}$. Set control input frequencies: if it falls below the cutoff frequency for a given pipeline and types of waves, the entered value is replaced with the value exceeding frequency cutoff at $0.01 \mathrm{GHz}$. The default values are set in accordance with article [1]. Feature at the point $r=0$ is the Laplace operator in the heat equation treated in the standard way: $\delta$ - function in the expression for the heat capacity was approximated by a step with half-width equal to $04^{\circ} \mathrm{C}$.
For the numerical analysis of the process of elimination of asphalt-paraffin corks was used the thermal parameters of high-paraffin oil: density oil and corks $\rho=$ $950 \mathrm{~kg} / \mathrm{m}^{3}$, specific heat capacity of oil and corks $C_{0}=$ $3 \mathrm{~kJ} /(\mathrm{kg} \mathrm{K})$, the phase transition temperature of $T_{S}=50^{\circ} \mathrm{C}$, the specific heat of melting $L=300 \mathrm{~kJ} / \mathrm{kg}$, thermal conductivity $\lambda=0,125 \mathrm{~W} /(\mathrm{m} \cdot \mathrm{K})$. Real and imaginary part of the dielectric constant $\varepsilon_{0}$ is chosen for dehydrated oil, in these cases, in a wide range of frequencies, these values are very weakly dependent on frequency, and in the calculations the typical values of the real $\varepsilon_{0}^{\prime} \approx 2.3$ and imaginary $\varepsilon_{0}^{\prime \prime} \approx 1.27610^{-}$parts of the complex permittivity of paraffin were used: ( $\varepsilon=2,3+i \cdot 0,01276$ ); the heat transfer coefficient (heat transfer from the external environment) from the end $x_{1}=$ $0.2 \mathrm{~W} /\left(\mathrm{m}^{2} \mathrm{~K}\right)$ and from the sides $\varkappa=1.613 \mathrm{~W} /\left(\mathrm{m}^{2} \mathrm{~K}\right)$, and the value of heat transfer coefficient on the lateral surface of the cylinder $\mathrm{r}=\mathrm{R}$ corresponds to the Nussle number $\mathrm{Nu}$ $=1$ (pipe in dry ground). The length of the paraffin plug is chosen equal to $\mathrm{H}=5 \mathrm{~m}$.

It was believed that the RF power of the electromagnetic wave is absorbed on the inner surface of the pipeline. Because a too small value of the length of penetration complicates the operational control of the movement of the source of electromagnetic radiation and because the depth of skin - layer is much less than the thickness of the pipe, it was believed that the wall thickness is equal to $4111111,110 \mathrm{Cm}$.

To study and analyze the nature of melting, configuration changes, temperature and speed of movement of the melting boundaries paraffin corks at different possible ambient temperatures $\left(0^{\circ} \mathrm{C}\right.$ to $\left.20^{\circ} \mathrm{C}\right)$ varied values of frequency and power source of electromagnetic radiation. Upon reaching the source of the upper boundary of the corks and if after a specified time interval, the calculation stops automatically.

Enter input parameters is carried out via a graphical interface. The parameters set in the program (including variable ones) include: a) characteristics of oil and paraffin plugs (densities, thermal conductivity coefficients, heat transfer coefficients, dielectric constant, specific heat of melting, ambient temperature, melting point); b) the parameters of the source of electromagnetic radiation (number of mode, conductivity of the pipeline walls, power of the source, frequency); c) the geometry of the tubing (radius, corks length, the distance from the radiator to the corks, the time interval); d) the parameters of the computational grid (the spacing/number of nodes along the radius, the interval/number of nodes along the radius, interval/number of nodes along the length of the corks, the time step (total time steps is calculated automatically depending on the magnitude of the time interval, the halfwidth of the Delta- function smoothing).

Data entry, management, and dynamic recalculation of the fields associated with the grid, are ensured.

1. Analyze the melting process of paraffin corks HF moving source with a capacity of $5,000 \mathrm{~kW}$ with a frequency of $1.4 \mathrm{GHz}$ at the ambient temperature $20{ }^{\circ} \mathrm{C}$. The first $41 \mathrm{~min}$. the paraffin layer is heated and heated to 
$50{ }^{0} \mathrm{C}$, the melt boundary starts to move inside the plug, and the phase boundary of the solid and liquid paraffin reaches the pipeline wall after 3 hours, and thus, as the plug penetrates ( 3 hours), the source begins to flow into the plug, The boundary of the melt (the boundary of the cork) was moved away from the source by a distance of $1.9 \mathrm{~m}$, this distance remains unchanged.

For frequency $\left(f=1.4 \cdot 10^{9} \mathrm{~Hz}\right)$ paraffin penetration corks coming from its center, which does the initial penetration of the corks. Progresses (center to $215{ }^{\circ} \mathrm{C}$ ) the source continues to move, and the boundary of the melt corks reaches a length of $5 \mathrm{~m}$ over a period of time of about 10 hours $(t=585 \mathrm{~min}$, Fig. 1.1), and the source reaches the border of the corks over $839 \mathrm{~min}$.

2. Let's investigate the process of melting paraffin plug, if the temperature of the external environment is less, namely: $10{ }^{\circ} \mathrm{C}$. The process of motion of the source occurs with a delay (with respect to item 1 ) for approximately 0.5 hours, the length from the source to the boundary of the melt also is 1.8 meters, and also the length of the plug is reached by the melt boundary with a delay of just over 0.5 hours and is $649 \mathrm{~min}$, the source itself will reach the end of the plug in 913 minutes.

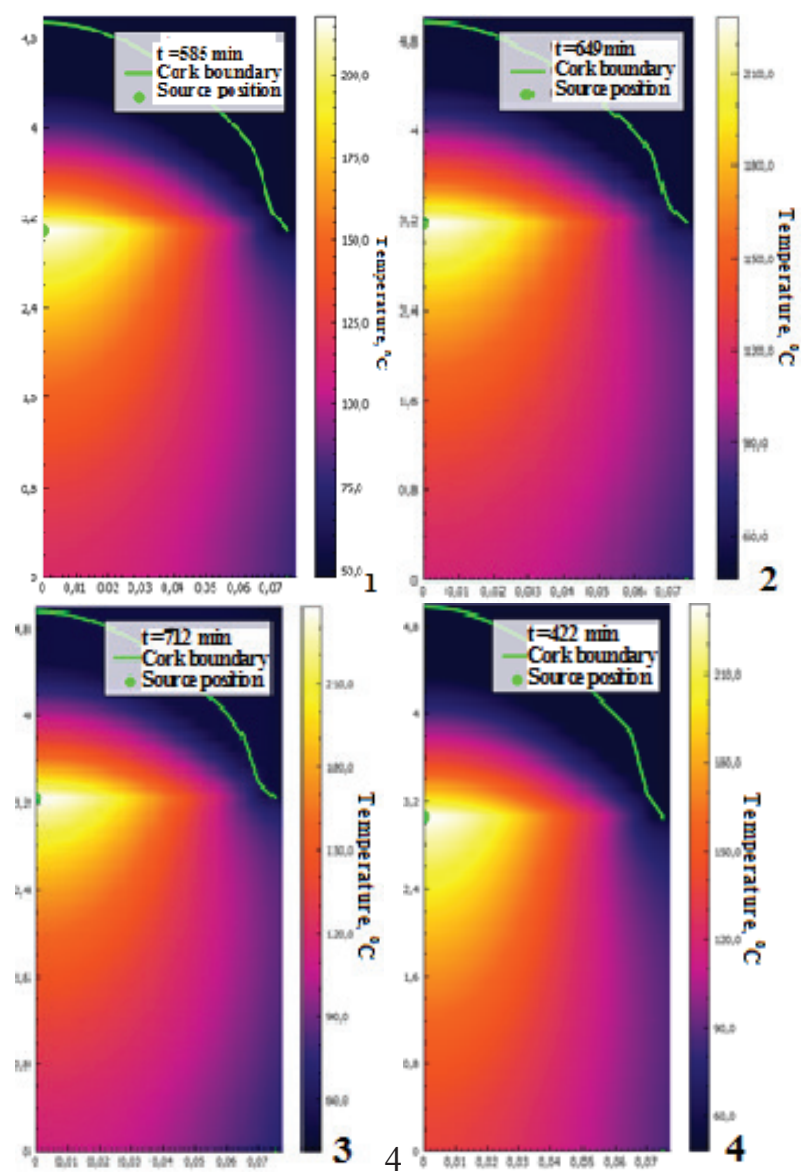

Fig. 1. Time melting of the plugs, temperature and configuration of the melt fuse for a frequency of $f=1.4 \cdot 10^{9} \mathrm{~Hz}$ when the power source $5000 \mathrm{~kW}$ at an external temperature 1) $20{ }^{\circ} \mathrm{C}$; 2) $10^{\circ} \mathrm{C}$; 3) $0{ }^{0} \mathrm{C}$ and 4) at the $\mathrm{t}=20^{\circ} \mathrm{C}$ at a power of $7000 \mathrm{~kW}$

3. If the temperature of the external medium drops to $0{ }^{\circ} \mathrm{C}$, the melt of the plug and the achievement of the melt boundary by the walls of the tube also occur with a delay of 3.5 hours, and at the same time starts the movement of the source into the corks (206 $\mathrm{min})$, the length from the source to the boundary of the melt is $1.75 \mathrm{~m}$. Initially, the boundary of the melt reaches the end of the corks $(5 \mathrm{~m})$ in the center over a period of time of more than 11.5 hours (712 minutes), the UPS continues to move forward and reach the end of the corks $(5 \mathrm{~m})$ for $986 \mathrm{~min}$. Temperature in the center (near the source) is $240{ }^{\circ} \mathrm{C}$.

Thus, with decreasing outside temperature, the source melts the paraffin plug for a longer time.

4. We will analyze the melting process of a paraffin plug with a moving RF source with an increased power to $7,000 \mathrm{~kW}$, but with the same frequency of $1.4 \mathrm{GHz}$ at an ambient temperature of $20^{\circ} \mathrm{C}$. Already after 0.5 hour (29 min) the melt boundary started to move and after 2 hours (132 $\mathrm{min}$ ) has reached the inner surface of the pipe, after which the source started to move deeper into the cork, and the distance from the source to the melt boundary was $1.95 \mathrm{~m}$. After 7 hours. (422 min), the melt boundary reached the end of the plug $(5 \mathrm{~m})$, and the source advanced to the end of the plug in 608 minutes. The temperature near the source was $230{ }^{\circ} \mathrm{C}$.

Times of melting of the corks ranges of temperature change and views of configuration of the cork melt for a frequency of $\mathrm{f}=1.4 \cdot 109 \mathrm{~Hz}$ with source capacity of 5000 $\mathrm{kW}$ at different external environment and at different capacities of the radiation source are presented in Fig. 1.(1 - 4).

5. Now consider the same process, only the power of the source was reduced to $3000 \mathrm{~kW}$ and all other parameters are left unchanged. Cork started to melt, about 68 minutes (a little more than 1 hour), and the source started moving source further deep into cork only after 283 $\min (\approx 5$ hour $)$. The distance from the source to the upper limit of the melt amounts to approximately a little less of $1.8 \mathrm{~m}$, and the source melting the entire length of the corks over a period of time more than 16 hours (975 minutes), that is, the lowering of the power source entails a sufficiently large increase in the time of melting of the corks of fixed length and a decrease in the speed of it melting. The source reaches the boundary of the corks only through $1372 \mathrm{~min}$ (slightly less than 25 hours). The temperature near the source does not exceed $200{ }^{0} \mathrm{C}$ $\left(185^{\circ} \mathrm{C}\right)$.

The configuration of the pattern boundary melting paraffin (geometry) with the source remains the same in all of these five studies, the difference is observed only in the temperature near the HF source and in the time of reaching the boundary of the when the melt boundary reaches the end of the corks.

6. Let us further investigate the influence of the change in the source frequency on the velocity, the characteristic shape (boundary geometry) of the melt, the time of melting of the corks and the temperature regime inside the pipeline. Analyze the melting process of paraffin corks HF moving source with a capacity of $5,000 \mathrm{~kW}$ with a frequency of 1.7 $\mathrm{GHz}$ at the ambient temperature $20{ }^{\circ} \mathrm{C}$. The movement of the melt boundary of the cork begins approximately after 1 hour (56 minutes) and after approximately 2.5 hours (154 minutes) the source starts to move along the length of the cork. The boundary of the melt is separated from the 
source by $1.2 \mathrm{~m}$ and has a more uniform power density distribution of heat over the cross section of the corks.

Localization of the initial melting is determined by the maximum power density of the heat $Q(r, z)$. Melting the entire length of the cork was carried out at $587 \mathrm{~min}$, and the source itself has moved to the end of the cork over 12 $\mathrm{h}(733 \mathrm{~min})$. The maximum temperature in the center of the melt was $130{ }^{\circ} \mathrm{C}$ (Fig. 2)

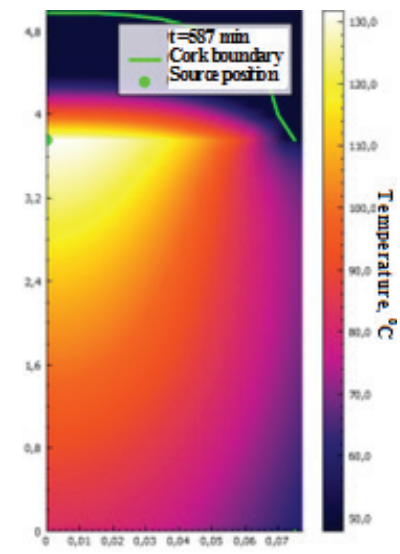

Fig. 2. The appearance of the border of the melt when reaching the end of its paraffin corks with a source frequency $f=1.7 \mathrm{GHz}$

7. Let us now consider what the situation will occur with the same frequency of $1.7 \mathrm{GHz}$, but at lower $\left(10{ }^{\circ} \mathrm{C}\right)$ ambient temperature. After 56 min started melting paraffin corks, also center of melting was at a distance of $0.04-0.05 \mathrm{~m}$ from the center of the corks (source), and upon reaching the melt of the boundary pipe, source has begun movement (through 121 minutes) at $84{ }^{\circ} \mathrm{C}$. The center of the border of the melt removed from the source center at a distance of about $0.65 \mathrm{~m}$, moving parallel with the source, reaches the boundaries of the paraffin cork through $562 \mathrm{~min}$, and the source goes to the end of the cork for $658 \mathrm{~min}$. The temperature in the center of the beginning of melting of paraffin plugs (located on concentric circles) does not exceed $92{ }^{\circ} \mathrm{C}$.

8. Next, change the frequency of the RF source, increasing it to $2.0 \mathrm{GHz}$ and leave all other parameters unchanged. First, the boundary melt paraffin plugs appears not in the center (where is the radiation source), but at a distance of about $0.04-0.05 \mathrm{~m}$ from the center of the radiation source. Here is the boundary of the melt reaches the maximum value, and then, this by heating boundary continues to move, and when the melt reaches the inner wall of the pipe, starts the motion of the source inside the pipe (Fig. 3). The initial distance from the source center to the center of the border of the melt amounts to little more than $0.65 \mathrm{~m}$. The temperature inside the pipe near the source is close to $90{ }^{\circ} \mathrm{C}$. After 2 hours, the source starts moving in the direction of the melting corks The boundary of the melt reaches the end of the cork not in its middle, but along the concentric circle at the same distance from the center of the emitter (i.e., distance from the center of the pipe is $0.04-0.05 \mathrm{~m}$ ) in $564 \mathrm{~min}$, while the source will move until the end of the corks in less than 11 hours (658 minutes).

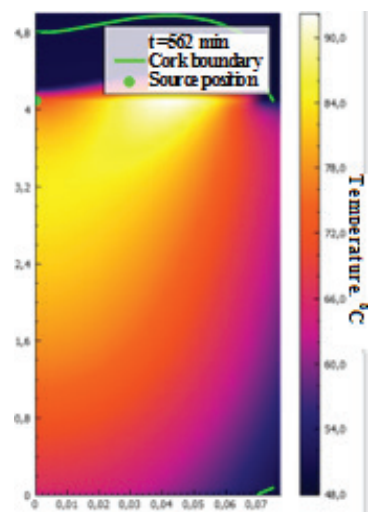

Fig. 3. The appearance of the border of the melt when reaching the end of its paraffin corks with a source frequency $f=2.0 \mathrm{GHz}$

9. If the ambient temperature will be more low $\left(10{ }^{\circ} \mathrm{C}\right)$, the overall picture of the geometry of the melt occurring at the same frequency of $2.0 \mathrm{GHz}$, does not change. The distance between centre of source and center of the border of melting of the cork remains the same (about $0.65 \mathrm{~m}$ ), the temperature in concentric circles melting is still not superior to $92{ }^{\circ} \mathrm{C}$, however, to achieve the boundary of the melt throughout the length of the corks required more time: $624 \mathrm{~min}$., and the source would will pass the whole length of the cork for 725 minutes, and the temperature of the initial melting of the boundaries of the corks will amount t o $98{ }^{\circ} \mathrm{C}$

10. Increasing further (Fig. 4) the frequency

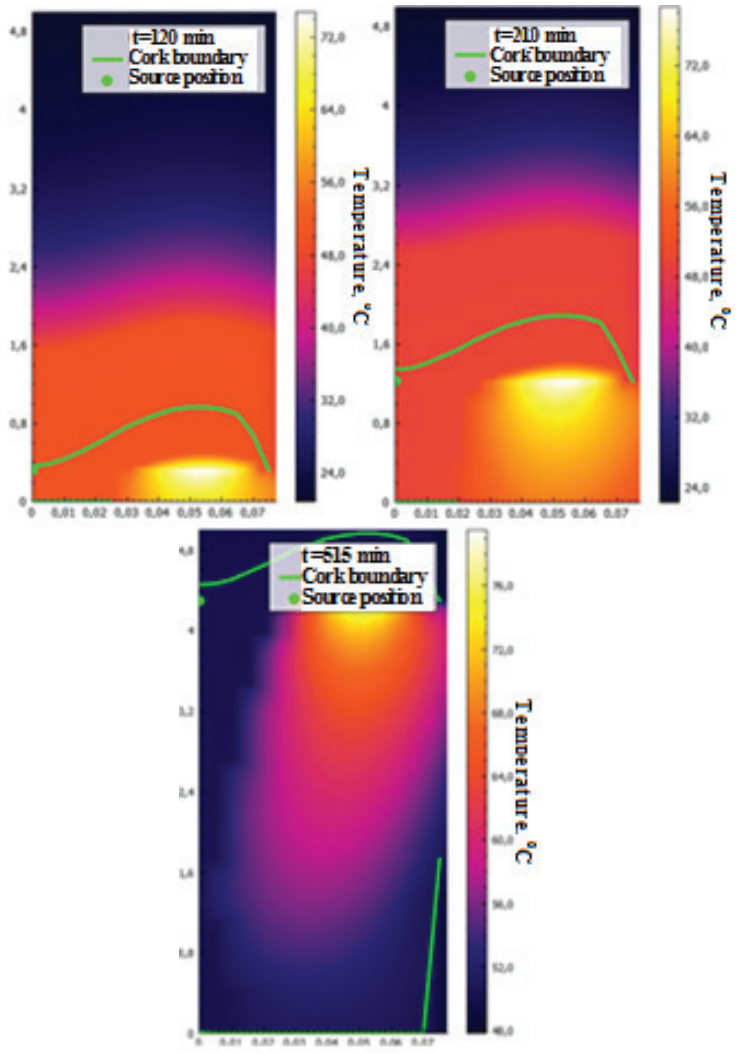

Fig. 4. The Appearance of the border of the melt and place of origin at different points of time at $f=2.5 \mathrm{GHz}$

of the RF source up to $2.5 \mathrm{GHz}$ at the unchanged all the other parameters, we note that the initial melting of the 
paraffin corks (at its maximum values) has the form of concentric circles (due to axial symmetry), the initial melting appears at a distance $0,054 \mathrm{~m}$ in $47 \mathrm{~min}$ and after about 1,5 hour ( $93 \mathrm{~min}$ ) reaches the inner surface of the pipeline, after which the source of HF energy starts moving, and the boundary of the melt in the center of the corks initially comes directly from the source and then (after $150 \mathrm{~min}$ ) the boundary of the melt is separated from the source, the distance from it increases gradually and at a distance of $0.2 \mathrm{~m}$ it stabilizes. After about 510 minutes ( 8.5 hours), the boundary reaches the end of the cork, still at a distance of about $0.054 \mathrm{~m}$ from the center of the pipeline, and the maximum temperature (in the concentric circle from the center of the pipeline) in the center of the melt does not exceed $78^{\circ} \mathrm{C}$

11. With further increase of the frequency of the RF source up to $3.0 \mathrm{GHz}$ with unaltered all the other parameters the initial melting of the corks occurs further away from the city center, approximately $0,058 \mathrm{~m}$ from the center of the pipeline (Fig. 5).

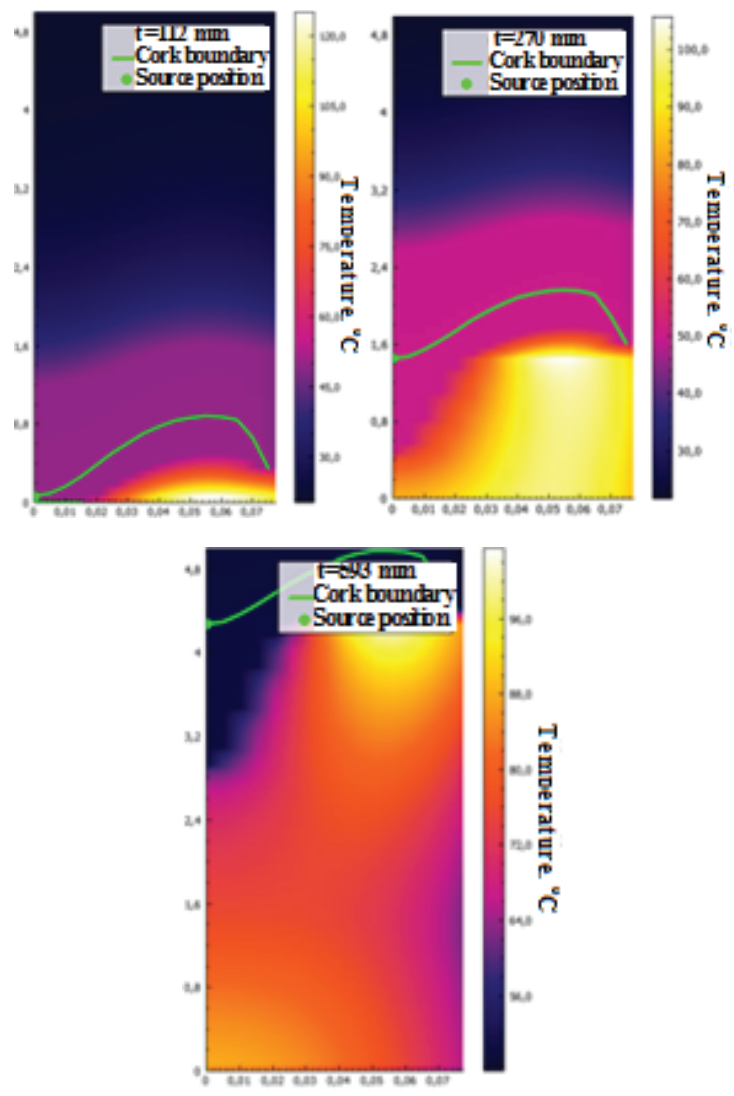

Fig. 5. The border of the melt and source at different points of time at $f=3.0 \mathrm{GHz}$

Through 593 min boundary reaches the end of the corks at the same distance $0,058 \mathrm{~m}$ from the center of the pipeline, moreover, the maximum temperature at the epicenter of the melt (obviously located on a concentric circle with a radius $0,058 \mathrm{~m}$ ) slightly increased, but, nevertheless, at the peak of the melt (in concentric circles) is not greater than $102{ }^{\circ} \mathrm{C}$.

The boundary of the melt comes from the source and moves with it, anywhere from it not coming off.

Thus, after the variation of these parameters of the RF source at different temperatures of the external environment, we can draw the following conclusions:

- Geometry and configuration of the boundaries of the melt corks depends only on the value of the source's frequency, and if the frequency is less than $2.0 \mathrm{GHz}$, the melting begins in the Central region. With increasing source frequency, the separation of the melt boundary from the source of RF radiation decreases, the boundary is smoothed

- Neither the ambient temperature nor the power of the radiation source does not have a significant impact on the geometry of the boundary of the melt. Change (increase or decrease) the power of the radiation source has an impact on the duration of melting of paraffin plugs (decrease and increase, respectively), the speed of the source itself (increase or decrease, respectively). The temperature inside the pipe is also affected by the external temperature: with a decrease in external temperatures, a longer warm-up time is required before the plug begins to melt, and the melting rate also decreases. When increasing (decreasing) power of the radiation source significantly increases (decreased) the temperature near the source, the time of melting decreases and Vice versa. As the temperature of the external environment also decreases the temperature near the center of the melting inside of the pipe.

- With an increase in frequency of more than 2.0 $\mathrm{GHz}$, the appearance of the melting surface changes significantly: the melting center is moved away from the center of the pipeline, turning into a concentric circle; the boundary of the melting is moved closer to the source (with increasing frequency) and at $\mathrm{f}=3.0 \mathrm{GHz}$ the boundary of the melting is attached to the source and does not detach from it.

- $\quad$ The temperature near the source at a frequency of more than $2.0 \mathrm{GHz}$ oscillates around $90{ }^{\circ} \mathrm{C}$, at $\mathrm{f}=2.5$ $\mathrm{GHz}$ this temperature is minimal (does not exceed $78{ }^{\circ} \mathrm{C}$ ), and then, with an increase of $\mathrm{f}=3.0 \mathrm{GHz}$, the temperature grows slightly, but not surpasses $102{ }^{\circ} \mathrm{C}$.

\section{References}

1. V. A. Balakirev, G. V. Sotnikov, Yu. V. Tkach, T. Yu. Yatsenko, ZhTF (Thech. Phys.) 71, 9 (2001)

2. A. A. Kislitsyn, R. I. Nigmatulin, J. Appl. Phys. (PMTF) 4 (1990)

3. R. I. Nigmatulin, K. M. Fedorov, Izv. Universities: Oil and gas, 10 (1991) 